\title{
THE POWER OF ECOMORPHY
}

NAPLES*, Virginia L., Department of Biological Sciences, Northern Illinois University, DeKalb, Illinois 60115-2861, U. S. A.;

MARTIN, Larry D., Department of Systematics and Ecology and Museum of Natural History, University of Kansas, Lawrence, Kansas 66045, U. S. A.

Similarity of structure among organisms may result from common inheritance or some common selective pressure. In the first case, the inherited features should vary no more between compared species than might be expected as individual variability within the common ancestor. In other words, the character should survive the most rigorous scrutiny. This does not mean that the character complex needs to have remained unchanged since the common ancestor, but only that those features which carry phylogenetic information are homologous in the strictest sense. Characters that result from convergent evolutionary innovations that are locally unique will usually fail a test of homology on anatomical and functional grounds. Convergent structures resulting from similarity of function, may be shared even when purpose differs. For example, many head motions seem to be shared by certain saber-toothed carnivores and rodents although the purpose of these motions, killing (saber-toothed carnivores) and digging (rodents) is quite different. When purpose is shared, characters group into integrated complexes that produce organisms of such broad similarity (ecomorphs) that they may become easily confused phylogenetically. We present a cladogram of carnivorous mammals showing the power of ecomorph adaptations to obscure phylogenetic history and point out the usefulness of functional and anatomical study independent of phylogenetic analysis as a means to illuminate this problem. 